\title{
Minimal polynomial descriptions of polyhedra and special semialgebraic sets
}

\author{
Gennadiy Averkov* and Ludwig Bröcker ${ }^{\dagger}$
}

November 1, 2018

\begin{abstract}
We show that a $d$-dimensional polyhedron $S$ in $\mathbb{R}^{d}$ can be represented by $d$-polynomial inequalities, that is, $S=\left\{x \in \mathbb{R}^{d}: p_{0}(x) \geq 0, \ldots, p_{d-1}(x) \geq 0\right\}$, where $p_{0}, \ldots, p_{d-1}$ are appropriate polynomials. Furthermore, if an elementary closed semialgebraic set $S$ is given by polynomials $q_{1}, \ldots, q_{k}$ and for each $x \in S$ at most $s$ of these polynomials vanish in $x$, then $S$ can be represented by $s+1$ polynomials (and by $s$ polynomials under the extra assumption that the number of points $x \in S$ in which $s q_{i}$ 's vanish is finite).
\end{abstract}

2000 Mathematics Subject Classification. Primary: 14P05, 52B11, 14Q99; Secondary: 52A20

Key words and phrases. Hörmander-Łojasiewicz's Inequality; polyhedron; polynomial; polytope; semialgebraic set; stability index; Theorem of Bröcker and Scheiderer

\section{Introduction}

Let $S \subseteq \mathbb{R}^{d}$ be a basic closed semialgebraic set, say

$$
S=\left\{x \in \mathbb{R}^{d}: p_{1}(x) \geq 0, \ldots, p_{k}(x) \geq 0\right\}=:\left\{p_{1} \geq 0, \ldots, p_{k} \geq 0\right\},
$$

where $p_{i} \in \mathbb{R}[X], X:=X_{1}, \ldots, X_{d}$.

It is known since the eighties that one can choose, for the description of $S$, polynomials $p_{1}, \ldots, p_{k}$ such that $k \leq \frac{d(d+1)}{2}$ (compare [Brö91]). Scheiderer [Sch89] gave examples showing that this bound is sharp. However, in these examples $S$ admits points $x$ where the local dimension of $S$ at $x$ is $m$ for all $1 \leq m \leq d$. So one might ask, if the bound for $k$ equals $d$ for sets $S$ of constant local dimension. In her diploma thesis A. Pauluhn Pau90 showed that for $d \in\{2,3\}$ equal dimensional basic closed sets can be characterized by at most 2 and 4 polynomials, respectively. All this holds true, if $\mathbb{R}$ is replaced by an arbitrary real closed field $R$. It seems that more is not known. Several authors (see Ber98, GH03, BGH05, Bos05) looked at the case where $S$ is a polytope, which might be interesting for applications (see also Hen07 for a survey on this topic). Also, they tried to find effective computations for suitable polynomials $p_{i}$ with $i=1, \ldots, s$, satisfying $S=\left\{p_{1} \geq 0, \ldots, p_{s} \geq 0\right\}$ starting from the description $S=\left\{l_{1} \geq 0, \ldots, l_{k} \geq 0\right\}$, where $l_{i}$ are linear forms (i.e., polynomials of degree at most one) and $k$ might be very large. Let $s=s(S)$ be the minimal possible value as above. One achieved the bound $s \leq 2 d-1$ in [BGH05. In GH03] one noticed that $s \geq d$ for polytopes and in BGH05] one conjectured that $s=d$ for $d$-dimensional polytopes. The equality $s=d$ was shown in Ber98 for polygons, in AH09a for simple polytopes and in AH09b] for three-dimensional polyhedra. The following two theorems are the main results of the manuscript. Theorem 1.1 below presents a short proof of a generalization of the result for simple polytopes from AH09a. Theorem 1.2 computes $s$ for all $d$-dimensional polyhedra.

Theorem 1.1. Let $S \subseteq \mathbb{R}^{d}$ be bounded and basic closed, say $S=\left\{q_{1} \geq 0, \ldots, q_{k} \geq 0\right\}$, where $q_{1}, \ldots, q_{k} \in$ $\mathbb{R}[X]$. Let $s \in \mathbb{N}$ be such that for each $x \in S$ there are at most $s$ polynomials $q_{i}$ among $q_{1}, \ldots, q_{k}$ where $q_{i}(x)=0$. Then the following statements hold.

*Fakultät für Mathematik, Universität Magdeburg, Universitätsplatz 2, D-39106 Magdeburg, email: averkov@ovgu.de

$\dagger$ Mathematisches Institut, Universität Münster, Einsteinstr. 62, D-48149, Münster, email: broe@math.uni-muenster.de 
a) $S=\left\{p_{1}, \ldots, p_{s+1} \geq 0\right\}$ for suitable $p_{1}, \ldots, p_{s+1} \in \mathbb{R}[X]$.

b) If there are only finitely many points $x_{1}, \ldots, x_{m} \in S$ where exactly $s$ polynomials $q_{i}$ vanish, then $S=\left\{p_{1} \geq 0, \ldots, p_{s} \geq 0\right\}$ for suitable $p_{1}, \ldots, p_{s} \in \mathbb{R}[X]$.

In view of Theorem 1.1, every simple $d$-dimensional polytope can be represented by $d$ polynomial inequalities. The above statement is also covered by the following

Theorem 1.2. Let $S$ be a d-dimensional polyhedron in $\mathbb{R}^{d}$. Let $k$ be the maximal dimension of an affine space contained in $S$. Then there exist $d-k$ polynomials $p_{0}, \ldots, p_{d-k-1}$ such that $S=\left\{p_{0} \geq\right.$ $\left.0, \ldots, p_{d-k-1} \geq 0\right\}$. Furthermore, $S$ cannot be represented by less than $d-k$ polynomials.

In Section 2 we present several separation theorems for semialgebraic sets. In Section 3 we prove Theorem 1.1. Finally, in Section 4 we prove Theorem 1.2

As in the above mentioned papers dealing with polynomial representations of polytopes, our work is semi-effective. That means, one has to check sequences of first order statements. As a consequence, in our theorems we can only compute the polynomials $p_{i}$ but one cannot bound their degrees in terms of the complexity of the "input polynomials". Equivalently, what we do below does not work over any real closed field.

We shall use the following notations. The Euclidean norm of $\mathbb{R}^{d}$ is denoted by $\|\cdot\|$. For the Euclidean topology of $\mathbb{R}^{d}$ we denote by cl, int, bd the closure, interior and boundary, respectively. We write $\mathrm{cl}^{Z}$ for the Zariski closure. Furthermore, the notations dim, aff, and relint stand for dimension, affine hull, and relative interior, respectively. For $x \in \mathbb{R}^{d}$ and $\rho>0$ let $B(x, \rho):=\left\{y \in \mathbb{R}^{d}:\|x-y\| \leq \rho\right\}$ and $\mathcal{U}(x, \rho):=\left\{y \in \mathbb{R}^{d}:\|x-y\|<\rho\right\}$.

We write as before $\left\{p_{1} \geq 0, \ldots, p_{k} \geq 0\right\}$ instead of $\left\{x \in \mathbb{R}^{d}: p_{1}(x) \geq 0, \ldots, p_{k}(x) \geq 0\right\}$ for polynomials $p_{1}, \ldots, p_{k} \in \mathbb{R}[X]$. Similarly we write $\left\{p_{1}>0, \ldots, p_{k}>0\right\}$ and $\left\{p_{1}=0, \ldots, p_{k}=0\right\}$. Note that $V:=\left\{p_{1}=0, \ldots, p_{r}=0\right\}=\{q=0\}$ for $q=p_{1}^{2}+\cdots+p_{r}^{2}$. $q$ is also called positive polynomial for the algebraic set $V$.

\section{Separation}

We shall use standard inequalities on continuous semialgebraic functions, see [BCR98, Section 2.6].

Theorem 2.1. Let $S \subseteq \mathbb{R}^{d}$ be a closed semialgebraic set and let $f, g, h$ be continuous semialgebraic functions on $S$ with $\{g=0\} \cap S \subseteq\{f=0\} \cap S$. Then there exists a positive polynomial $p$ and $N \in \mathbb{N}$ such that $\left|f^{N} h\right| \leq|p g|$ on $S$.

The version for $h(X)=1$ follows from the results in [BCR98, Section 2.6]. The version for $h(X)=1$ obviously implies the version for a general $h$. For the special case that $S$ is bounded and $h(X)=1$ we may obviously choose $p$ to be a constant, which yields the well-known Hörmander-Eojasiewicz Inequality. The polynomial $p$ can be chosen to have a specific form $p(X)=\left(1+\|X\|^{2}\right)^{M}$ with $M \in \mathbb{N}$. Some consequences of Theorem 2.1 (and, more specifically, Hörmander-Łojasiewicz's Inequality) will be useful in our subsequent derivations.

Proposition 2.2. Let $A$ be an unbounded, closed semialgebraic set and $f, g$ be continuous semialgebraic functions on $A$ such that $f>0, g \geq 0$ and $f(x) \rightarrow \infty$ as $x \in A$ and $x \rightarrow \infty$. Then there exists $\gamma>0$ and $N \in \mathbb{N}$ such that $\gamma f^{N} \geq g$ on $A$.

Proof. Let $A=A_{0} \cup A_{1}$, where $A_{0}, A_{1}$ are semialgebraic, closed, $A_{0}$ is bounded, $o \notin A_{1}$ and $f \geq 2$ on $A_{1}$.

By Theorem 2.1, $g \leq \alpha\left(1+\|X\|^{2}\right)^{M}$ on $A$ for appropriate $\alpha>0$ and $M \in \mathbb{N}$. The inequality

$$
f^{N} \geq \alpha\left(1+\|X\|^{2}\right)^{M}
$$

is fulfilled on $A_{1}$ if

$$
f\left(\frac{X}{\|X\|^{2}}\right)^{-N} \leq \frac{\|X\|^{2 M}}{\alpha\left(\|X\|^{2}+1\right)^{M}}
$$


for all $X$ with $\frac{X}{\|X\|^{2}} \in A_{1}$. Let $\tilde{A}_{1}:=\{o\} \cup\left\{x \in \mathbb{R}^{d} \backslash\{o\}: \frac{x}{\|x\|^{2}} \in A_{1}\right\}$ and $\tilde{f}$ a semialgebraic function on $\tilde{A}_{1}$ given by

$$
\tilde{f}(x):= \begin{cases}f\left(\frac{X}{\|X\|^{2}}\right)^{-1} & \text { if } x \in \tilde{A}_{1} \backslash\{o\}, \\ 0 & \text { if } x=0 .\end{cases}
$$

Clearly, $\tilde{A}_{1}$ is bounded and closed. Thus, for verification of (2.1) we need to show

$$
\tilde{f}^{N} \leq \frac{\|X\|^{2 M}}{\alpha\left(\|X\|^{2}+1\right)^{M}} \quad \text { on } \tilde{A}_{1} .
$$

The existence of $N$ satisfying the above relation follows from Łojasiewicz's inequality (by taking into account that $\tilde{f} \leq \frac{1}{2}$ on $\tilde{A}_{1}$ ). The assertion follows by defining a $\gamma>1$ such that $\gamma f^{N} \geq g$ on $A_{0}$.

Definition 2.3. Let $S, T \subseteq \mathbb{R}^{d}$ be semialgebraic sets and let $p \in \mathbb{R}[X]$. We say that $p$ separates $S$ from $T$ if $p \geq 0$ on $S, p \leq 0$ on $T$, and $\{p=0\} \cap(S \cup T) \subseteq \mathrm{cl}^{Z}(S \cap T)$.

The polynomials from Lemma 2.4 will be frequently used in our constructions.

Lemma 2.4. Let $0<\delta<\rho$ and $m \in \mathbb{N}$. Then the following two statements hold true.

a) There exists a polynomial $\kappa=\kappa_{\delta, \rho, m} \in \mathbb{R}[t]$ such that

i) $\kappa \geq 0$ on $[-\rho, \rho]$ and $\{\kappa=0\} \cap[-\rho, \rho]=\{0\}$,

ii) $\kappa \leq \frac{1}{4^{m}}$ on $[-\rho, 0]$,

iii) $\kappa \geq 2$ on $[\delta, \rho]$,

iv) $\kappa \leq 3$ on $[0, \rho]$.

b) There exists a polynomial $\mu:=\mu_{\delta, \rho} \in \mathbb{R}[t]$ such that

i) $\mu>0$ on $[-\rho, \infty[$,

ii) $\mu<\frac{1}{2}$ on $[-\rho, 0]$,

iii) $\mu>2$ on $[\delta, \infty[$.

In particular, $\mu(t) \rightarrow \infty$ for $t \rightarrow \infty$

Proof. (a) Consider a continuous function $\phi:[-\rho, \rho] \rightarrow \mathbb{R}$ such that $\frac{1}{4^{m+1}} \leq t^{2} \phi(t) \leq \frac{1}{2 \cdot 4^{m}}$ for $t \in[-\rho, 0]$, $t^{2} \phi(t) \geq 2+\frac{1}{3}$ for $t \in[\delta, \rho]$ and $t^{2} \phi(t) \leq 3-\frac{1}{3}$ for $t \in[0, \rho]$. Now by explicit Stone-Weierstrass Approximation (cf. [BCR98, Theorem 8.8.5]) of $\phi(t)$ by a polynomial $\pi(t)$ we get $\kappa(t)=t^{2} \pi(t)$.

b) We set

$$
\mu_{\delta, \rho}(t):=\left(\frac{t+2 \rho-\delta / 2}{2 \rho}\right)^{2 k}
$$

where $k \in \mathbb{N}$ is sufficiently large.

Proposition 2.5. (Separation of disjoint closed sets) Let $S, T \subseteq \mathbb{R}^{d}$ be closed semialgebraic sets such that $S \cap T=\emptyset$. Let $S$ be compact and basic closed, say $S=\left\{f_{1} \geq 0, \ldots, f_{k} \geq 0\right\}$, where $f_{1}, \ldots, f_{k} \in \mathbb{R}[X]$. Then there exists a polynomial $p \in \mathbb{R}[X]$ which separates $S$ from $T$.

Proof. We define the polynomial mapping $F(X):=\left(f_{1}(X), \cdots, f_{k}(X)\right)$ from $\mathbb{R}^{d}$ to $\mathbb{R}^{k}$. Then $F(S) \subseteq \mathbb{R}_{>0}^{k}$ and $F(T) \subseteq \mathbb{R}^{k} \backslash \mathbb{R}_{\geq 0}^{k}$ are compact resp. closed semialgebraic sets. Choose $\rho>0$ such that $F(S) \subseteq[0,2 \rho]^{k}$. We define the polynomial $g_{m}(Y):=\rho^{2 m} k+\frac{1}{m}-\sum_{i=1}^{k}\left(Y_{i}-\rho\right)^{2 m}$ in indeterminates $Y:=Y_{1}, \ldots, Y_{k}$, where $m \in \mathbb{N}$. The semialgebraic set $G_{m}:=\left\{g_{m} \geq 0\right\} \subseteq \mathbb{R}^{k}$ approximates $[0,2 \rho]^{k}$ with any given precision (in the Hausdorff metric), as $m \rightarrow \infty$. Moreover, $[0,2 \rho]^{k}$ is contained in the interior of $G_{m}$ for every $m \in \mathbb{N}$. Consequently, $G_{m}$ is disjoint with $F(T)$ if $m$ is sufficiently large. Hence we may define $p:=g_{m} \circ F$ with $m$ sufficiently large. 
Remark 2.6. Proposition 2.5 still holds, if $S$ is not necessarily basic. Also, it can be shown directly by Stone-Weierstrass Approximation, but the way we did it is more constructive.

Proposition 2.7. (Globalizing a local separator) Let $S \subseteq \mathbb{R}^{d}$ be bounded and closed semialgebraic set and let $T \subseteq \mathbb{R}^{d}$ be a closed semialgebraic set. Let $r \in \mathbb{R}[X]$ be such that $r \geq 0$ on $\mathbb{R}^{d}, S \cap T=\{r=0\}$ and $r(X) \rightarrow \infty$ as $\|X\| \rightarrow \infty$. Let $f \in \mathbb{R}[X]$ be such that $f$ separates $S \cap U$ from $T \cap U$ for an open, semialgebraic set $U$ with $S \cap T \subseteq U$. Then there exists a polynomial $p$ separating $S$ from $T$.

Proof. Since $r(x) \rightarrow \infty$ as $\|x\| \rightarrow \infty$, the set $\{r \leq \varepsilon\}$ is bounded for every $\varepsilon \geq 0$. If $0<\varepsilon \leq \frac{1}{2}$ is small enough, then $\{r \leq \varepsilon\} \subseteq U$. In view of Proposition 2.5, there exists a polynomial $g$ that separates $S \cap\left\{r \geq \frac{\varepsilon}{4}\right\}$ from $T \cap\left\{r \geq \frac{\varepsilon}{4}\right\}$. We define $p:=f+\left(\frac{2 r}{\varepsilon}\right)^{N} g$ with $N \in \mathbb{N}$ to be chosen below. In view of Lojasiewicz's inequality we have $\frac{1}{2}|f| \geq\left|\frac{2 r}{\varepsilon}\right|^{N}|g|$ on $(S \cup T) \cap\left\{r \leq \frac{\varepsilon}{4}\right\}$ for all sufficiently large $N$. On the other hand, by Proposition 2.2, $\frac{1}{2}\left|\frac{2 r}{\varepsilon}\right|^{N}|g| \geq|f|$ on $(S \cup T) \cap\{r \geq \varepsilon\}$ for all sufficiently large $N$. Thus, $p$ separates $S$ from $T$ if $N$ is sufficiently large.

Proposition 2.8. (Merging local separators) Let $S, T \subseteq \mathbb{R}^{d}$ be closed semialgebraic sets and let $x_{1}, \ldots, x_{m} \in$ $\mathbb{R}^{d}$. Assume that there exists $\rho>0$ such that for every $i \in\{1, \ldots, m\}$ one has $\left\{x_{i}\right\}=S \cap T \cap B\left(x_{i}, \rho\right)$ and one can find a polynomial $p_{i}$ separating $S \cap B\left(x_{i}, \rho\right)$ from $T \cap B\left(x_{i}, \rho\right)$. Then there exist $\rho>0$ and $p \in \mathbb{R}[X]$ such that $p$ separates $S \cap U$ from $T \cap U$ for $U:=\bigcup_{i=1}^{m} B\left(x_{i}, \rho\right)$.

Proof. Let $r_{i}:=\left\|X-x_{i}\right\|^{2}$. Choose $\rho>0$ to be small enough and a $\delta$ such that $\rho<\delta<\left\|X_{i}-X_{j}\right\|-\rho$ for $1 \leq i<j \leq m$. Then, by Lojasiewicz's inequality, for a sufficiently large $N \in \mathbb{N}$ one has $\left(\frac{r_{i}}{\delta^{2}}\right)^{N} \leq \frac{1}{2}\left|p_{i}\right|$ on $(S \cup T) \cap B\left(x_{i}, \rho\right)$ and $p_{i}+\left(\frac{r_{i}}{\delta^{2}}\right)^{N}>0$ on $B\left(x_{j}, \rho\right)$ for $1 \leq i<j \leq m$. Thus, we may choose

$$
p:=\prod_{i=1}^{m}\left(p_{i}+\left(\frac{r_{i}}{\delta^{2}}\right)^{N}\right) .
$$

The following proposition is a straightforward consequence of Propositions 2.7 and 2.8 .

Proposition 2.9. (Separation of sets with finite intersection) Let $S, T \subseteq \mathbb{R}^{d}$ be semialgebraic sets, $T$ closed, $S$ basic closed and bounded, such that $S \cap T$ is finite, say $S \cap T=\left\{x_{1}, \ldots, x_{m}\right\}$. Then there exists a polynomial $p$ which separates $S$ from $T$ if and only if this holds locally, that means: There exists $\rho>0$ such that for $i \in\{1, \ldots, m\}$ there exists a polynomial $p_{i}$ which separates $B\left(x_{i}, \rho\right) \cap S$ from $B\left(x_{i}, \rho\right) \cap T$.

Remark 2.10. The following generalization of Proposition 2.9 holds true. Let $S, T \subseteq \mathbb{R}^{d}$ be semialgebraic, $S$ compact, $T$ closed. Then there exists a polynomial $p$ which separates $S$ from $T$ if and only if for any finite set of points $X$ there exists $\rho>0$ and a polynomial $q$ such that $q$ separates $S \cap U$ from $T \cap U$ for $U:=\left(\bigcup_{x \in X} B(x, \rho)\right)$. This follows from Proposition 6.10 (Chapter VI) in ABR96, whose proof, however, is not at all constructive.

Proposition 2.11. Let $S, T_{1}, T_{2} \subseteq \mathbb{R}^{d}$ be semialgebraic sets, where $S$ is basic closed, $T_{1}$ is compact and $T_{2}$ is closed. Let $h$ be a non-negative polynomial with $\{h=0\}=\operatorname{cl}^{Z}\left(S \cap T_{1}\right)$ and let $f \in \mathbb{R}[X]$ be an arbitrary polynomial. Assume that

a) $S \cap T_{2}=\emptyset$,

b) $\operatorname{int}\left(S \cap T_{1}\right)=\emptyset$,

c) $h>0$ on $T_{2}$ and, for $x \in T_{2}, h(x) \rightarrow \infty$, as $\|x\| \rightarrow \infty$.

d) $\{f=0\} \cap T_{1} \subseteq S \cap T_{1}$.

Then there exists $p \in \mathbb{R}[x]$ such that

i) $p \geq f$ on $S$,

ii) $\operatorname{sign} p \leq \operatorname{sign} f$ on $T_{1}$, 
iii) $p<0$ on $T_{2}$.

Proof. By assumption (c) there exists $\alpha>0$ such that $h \geq \alpha$ on $T_{2}$. Choose $\delta$ with $0<2 \delta<\alpha$ and $\delta<\rho$. We introduce the polynomial $\mu=\mu_{\delta, \rho}$ as in Lemma 2.4. b). Let $T_{0}:=T_{1} \cap\{h \geq \delta\}$. Then $S \cap T_{0}=\emptyset$. Taking into account assumption 國) and applying Proposition 2.5, we can find a polynomial $q \in \mathbb{R}[X]$ with $q>0$ on $S$ and $q<0$ on $T_{0} \cup T_{2}$. Now, set $p:=f+q \cdot h^{l} \cdot[\mu \circ(h-\delta)]^{l+m}$, where $l, m \in \mathbb{N}$ are sufficiently large.

\section{Small polynomial description of special semialgebraic sets}

If $a_{1}, \ldots, a_{k} \in \mathbb{R}$, let $\sigma_{i}\left(a_{1}, \ldots, a_{k}\right)$ denote the $i$ th elementary symmetric function of $a_{1}, \ldots, a_{k}$, that is,

$$
\sigma_{i}\left(a_{1}, \ldots, a_{k}\right):=\sum_{1 \leq j_{1}<\ldots<j_{i} \leq k} a_{j_{1}} \cdots a_{j_{i}} .
$$

The basic observation is the following.

Lemma 3.1. Let $\rho>0$ and $s, k \in \mathbb{N}$ with $s \leq k$. Then there exists $\varepsilon>0$ such that for all $a_{1}, \ldots, a_{k} \in \mathbb{R}$ with $\left|a_{1}\right| \leq \varepsilon, \ldots,\left|a_{s}\right| \leq \varepsilon$ and $a_{s+1} \geq \rho, \ldots, a_{k} \geq \rho$ one has $a_{1}, \ldots, a_{s} \geq 0$ if and only if

$$
\sigma_{k-s+1}\left(a_{1}, \ldots, a_{k}\right) \geq 0, \ldots, \sigma_{k}\left(a_{1}, \ldots, a_{k}\right) \geq 0 .
$$

Proof. The necessity is trivial. Let us prove the sufficiency. Consider the polynomial

$$
\prod_{i=1}^{k}\left(t+a_{i}\right)=\sum_{i=1}^{k} \sigma_{i}\left(a_{1}, \ldots, a_{k}\right) t^{k-i}+t^{k}
$$

For $i \leq k-s$ there is a summand in the definition of $\sigma_{i}\left(a_{1}, \ldots, a_{k}\right)$ without factors belonging to $\left\{a_{1}, \ldots, a_{s}\right\}$. The summands containing a factor of $\left\{a_{1}, \ldots, a_{s}\right\}$ tend to zero for $\varepsilon \rightarrow 0$. Hence for all sufficiently small $\varepsilon>0$ depending on $k, s, \rho$ and for $i \leq k-s$ one has $\sigma_{i}\left(a_{1}, \ldots, a_{k}\right)>0$. One has $\sigma_{i}\left(a_{1}, \ldots, a_{k}\right) \geq 0$ for $i>k-s$, by the assumption. Consequently, the polynomial $\prod_{i=1}^{k}\left(t+a_{i}\right)$ has no positive roots, which implies that $a_{1} \geq 0, \ldots, a_{k} \geq 0$.

Proof of Theorem 1.1. 目) Let $p_{i}:=\sigma_{k-s+i}\left(q_{1}, \ldots, q_{k}\right)$ for $i \in\{1, \ldots, s\}$. Then $S \subseteq\left\{p_{1} \geq 0, \ldots, p_{s} \geq 0\right\}$. Moreover, by Lemma 3.1. for each $x \in \mathrm{bd} S$ there is a neighborhood $B(x, \varepsilon)$ such that $S \cap B(x, \varepsilon)=$ $\left\{p_{1} \geq 0, \ldots, p_{s} \geq 0\right\} \cap B(x, \varepsilon)$. That means

$$
\left\{p_{1} \geq 0, \ldots, p_{s} \geq 0\right\}=S \cup T,
$$

where $T \subseteq \mathbb{R}^{d}$ is semialgebraic, closed and

$$
T \cap S=\emptyset .
$$

Now, according to Proposition 2.5] we can choose $p_{s+1} \in \mathbb{R}[X]$ which separates $S$ from $T$.

b) We take $p_{1}, \ldots, p_{s}$ as before. Let $\left\{x_{1}, \ldots, x_{m}\right\}$ be the set of all points in $S$ where exactly $s$ polynomials $q_{i}$ vanish. We see that $R:=\left(\left\{p_{1} \geq 0, \ldots, p_{s-1} \geq 0\right\} \backslash S\right) \cup\left\{x_{1}, \ldots, x_{m}\right\}$ is closed. Clearly, $R \cap S=\left\{x_{1}, \ldots, x_{m}\right\}$. Moreover, for each $x_{i}$ there is a ball $B(x, \varepsilon)$ such that $p_{s}$ separates $B\left(x_{i}, \varepsilon\right) \cap S$ and $B\left(x_{i}, \varepsilon\right) \cap R$. Now, according to Proposition 2.9 we can modify $p_{s}$ to a polynomial separates $S$ from $R$.

Remark 3.2. Analogous semi-effective results can also be obtained for basic open sets by using similar methods. 


\section{Minimal description of polyhedra}

A subset of $\mathbb{R}^{d}$ is said to be a polyhedron if it is the intersection of finitely many closed halfspaces. Bounded polyhedra are called polytopes. For background information on polytopes and polyhedra we refer to Zie95. By $\mathcal{F}(P)$ we denote the set of all faces of $P$. By $\mathcal{F}_{k}(P)$ we denote the set of all $k$ dimensional subfaces of $P$. If the choice of $P$ is clear we merely write $\mathcal{F}_{k}$ and $\mathcal{F}$. Polytopes of dimension $d$ are called $d$-polytopes. Faces of dimension 0 and $\operatorname{dim} P-1$ are called vertices and facets, respectively. We introduce the $k$-skeleton (also called the set of all $k$-extremal points) of $P$ by

$$
\operatorname{ext}_{k} P:=\bigcup_{F \in \mathcal{F}_{k}(P)} F
$$

We also write ext $P:=\operatorname{ext}_{0} P$.

Notations and Remarks 4.1. Let $S \subseteq \mathbb{R}^{d}$ be a polyhedron.

a) For a $k$-face $F$ of $S$ we fix a degree-one polynomial $l_{F}$ such that $F=\left\{l_{F}=0\right\} \cap P$ and $l_{F} \geq 0$ on $S$. Then

$$
D_{k}(S):=\bigcap_{F \in \mathcal{F}_{k}}\left\{l_{F} \geq 0\right\}
$$

is called a $k$-support of $S$. The $k$-support depends on the choice of $l_{F}$ 's. We have $D_{d-1}(S)=S$.

b) For $l<k$ every $l$-face of $S$ is also an $l$-face of $D_{k}(S)$.

c) If $S$ is compact, $D_{k}(S)$ is not compact in general, unless $l_{F}$ 's are chosen in a suitable way.

d) For a vertex $x \in S$ we set

$$
S_{x}:=\bigcap_{\substack{F \in \mathcal{F}_{d-1}(S) \\ x \in F}}\left\{l_{F} \geq 0\right\} \quad \text { and } \quad S_{x}^{*}:=\bigcap_{\substack{F \in \mathcal{F}_{d-1}(S) \\ x \notin F}}\left\{l_{F}>0\right\} .
$$

Notice that $P_{x}$ is closed and $P_{x}^{*}$ is open.

e) Now let $S$ be a polytope. Then there exists a sequence of $k$-supports $D_{-1}(S), \ldots, D_{d-1}(S)$ with

$$
S=D_{d-1}(S) \subseteq \cdots \subseteq D_{0}(S) \subseteq D_{-1}(S)=\mathbb{R}^{d} .
$$

and $D_{k}(S) \backslash \operatorname{ext}_{k-1} S \subseteq \operatorname{int}\left(D_{k-1}\right)$ for $k=0, \ldots, d-1$.

f) For $k=0, \ldots, d-1$ one has

i) $D_{k}(S)=\bigcap_{x \in \operatorname{ext} S} D_{k}(S)_{x}$.

ii) There is a compact semialgebraic set $R_{k}$ such that

$$
S \subseteq \operatorname{int}\left(R_{k}\right) \subseteq R_{k} \subseteq \bigcup_{x \in \operatorname{ext} S} D_{k}(S)_{x}^{*}
$$

Here i) holds, since each facet of $D_{k}(S)$ contains a vertex of $S$, and ii) follows from the inclusion

$$
S \subseteq \bigcup_{x \in \operatorname{ext} S} D_{k}(S)_{x}^{*}
$$

Let us show (4.1). Take an arbitrary $y \in S$. Then there exists a unique face $G$ with $y \in \operatorname{relint} G$. Let $x$ be any vertex of $G$ and let $F$ be a facet with $x \notin F$. Then $y \notin F$, since otherwise we would have relint $G \cap F \neq \emptyset$, which implies $G \subseteq F$ and by this $x \in F$, a contradiction. Hence $l_{F}(y)>0$. This yields (4.1).

Proposition 4.2. For $k=0, \ldots, d-1$ there is a polynomial $p_{k}$ such that 
a) $p_{k} \geq 0$ on $S$

b) $p_{k} \leq 0$ on $D_{k-1}(S) \backslash \operatorname{int}\left(D_{k}(S)\right)$

c) $\left\{p_{k}=0\right\} \cap\left(D_{k-1}(S) \backslash \operatorname{int}\left(D_{k}(S)\right)\right) \subseteq S$

In (b) and (C) we could also replace $D_{k-1}(S) \backslash \operatorname{int}\left(D_{k}(S)\right)$ by $D_{k-1}(S) \backslash D_{k}(S)$, but for the proof by induction, which we give below, it is better to have it this way.

Proof of Proposition 4.2. The proof is by induction on $d$. For starting the induction argument we consider the cases $k=0$ and $k=d-1$ separately, which is done in Steps 1 and 2 of the proof. In the remaining steps we apply the inductive assumption to all vertex figures of $S$. Appropriately combining the polynomials associated to the vertex figures we generate polynomials associated to $S$. In Step 3 for each vertex of $S$ we construct a polynomial satisfying a)-c) in a small neighborhood of that vertex. We combine these polynomials in Step 6, thus getting a polynomial $r_{k}$ and show in Step 7 that $r_{k}$ fulfills the conditions a),b),c) locally, that means in a set $Q_{k-1}$ which we get by restriction to $R_{k}$. This is the main step. It uses decompositions of $Q_{k-1}$ which are explained in Step 4 and 5. Finally, in Step 8 we globalize $r_{k}$ in order to get $p_{k}$.

Step 1: $k=0$. We need to show the existence of $p_{0}$ such that $p_{0} \geq 0$ on $S, p_{0} \leq 0$ on $\mathbb{R}^{d} \backslash \operatorname{int} D_{0}(S)$ and $\left\{p_{0}=0\right\} \cap\left(\mathbb{R}^{d} \backslash \operatorname{int} D_{0}(S)\right) \subseteq S$. We use Proposition 2.9, where $T:=\mathbb{R}^{d} \backslash \operatorname{int}\left(D_{0}(S)\right)$. Then $S \cap T=\operatorname{ext} S$ is a finite set, and a local separation of $S$ from $T$ around each $x \in S \cap T$ can be achieved.

Step 2: $k=d-1$. Let $\mathcal{F}_{d-1}(S)=\left\{F_{1}, \ldots, F_{r}\right\}$. We define $p_{d-1}:=\prod_{i=1}^{r} l_{F_{i}}$. Clearly, $p_{d-1} \geq 0$ on $S$. Now let

$$
S_{i}:=\left\{l_{F_{i}} \leq 0\right\} \cap \bigcap_{\substack{j=1, \ldots, r \\ j \neq i}}\left\{l_{F_{j}} \geq 0\right\} .
$$

Then by construction we have $D_{d-2}(S) \subseteq \bigcup_{i=1}^{r} S_{i}, p_{d-1}<0$ on $\left(\bigcup_{i=1}^{r} S_{i}\right) \backslash S$ and $p_{d-1}=0$ on bd $S$. This proves b) and c).

The case $d=1$ is trivial. Cases 1 and 2 yield the assertion for $d=2$. Now assume that $d \geq 3$.

Step 3: Construction of a local solution $q_{i, k}$. Let ext $S=\left\{x_{0}, \ldots, x_{m}\right\}$. For small $\varepsilon>0$ the hyperplane $\left\{l_{x_{i}}=\varepsilon\right\}$ intersects $S$, say $S^{i}:=\left\{l_{x_{i}}=\varepsilon\right\} \cap S$, where we choose $\varepsilon$ such that $l_{x_{i}}\left(x_{j}\right)>\varepsilon$ for $j \neq i$. Also, we set $D_{i, k-1}=\left\{l_{x_{i}}=\varepsilon\right\} \cap D_{k}(S)$. So, we can use the inductive assumption to the $(d-1)$-dimensional polytope $S^{i}$ (in the $(d-1)$-dimensional affine space aff $S^{i}$ ). For all $\varepsilon>0$ as above $S^{i}$ remains the same up to a homothety. Let $1 \leq k \leq d-2$ be given. We want to construct a suitable polynomial $p_{k}$. For $i=1, \ldots, m$ let $p_{i, k-1}$ be a polynomial as in the assertion with respect to the polytope $S^{i}$ and the sets $D_{i, k-1}$ and $D_{i, k-2}$. Now let $q_{i, k}$ be the homogenization of $p_{i, k}$ with respect to the center $x_{i}$. Around $x_{i}$ the polynomial $q_{i, k}$ satisfies the properties a)-c). So, in order to generate $p_{k}$ we should combine the polynomials $q_{i, k}$ in a suitable way.

Step 4: Notations and Remarks. For $k=0, \ldots, d-1$ let

$$
Q_{k-1}:=R_{k} \cap\left(D_{k-1}(S) \backslash \operatorname{int}\left(D_{k}(S)\right)\right)
$$

Then $Q_{k-1}$ is compact. Let $G_{1}, \ldots, G_{s}$ be the facets of $D_{k}(S)$. (Here we keep $k$ fixed, so we omit the index $k$ at the $G_{i}$ 's.) For $\delta>0$ and $x_{i} \in S_{0}=\left\{x_{1}, \ldots, x_{m}\right\}$ let

$$
D_{k}(S)_{x_{i}, \delta}^{*}:=\bigcap_{\substack{j=1, \ldots, s \\ x_{i} \notin G_{j}}}\left\{l_{G_{j}} \geq \delta\right\}
$$

We have

$$
D_{k}(S)_{x_{i}, \delta}^{*} \subseteq D_{k}(S)_{x_{i}}^{*} \subseteq \operatorname{cl} D_{k}(S)_{x_{i}}^{*}=\bigcap_{\substack{j=1, \ldots, s \\ x_{i} \notin G_{j}}}\left\{l_{G_{j}} \geq 0\right\} .
$$

By Remark 4.1f)ii) there exists a $\delta>0$ such that

$$
Q_{k-1} \subseteq \bigcup_{i=1}^{m} D_{k}(S)_{x_{i}, \delta}^{*}
$$


Step 5: Notations and Remarks. For every decomposition $\{1, \ldots, m\}=\{i\} \cup A \cup B$ let

$$
\begin{aligned}
Q_{k-1}^{+}(A) & :=\bigcap_{\alpha \in A} Q_{k-1} \cap D_{k}(S)_{x_{\alpha}}^{*}, \\
Q_{k-1}^{-}(B) & :=\bigcap_{\beta \in B} Q_{k-1} \backslash D_{k}(S)_{x_{\beta}}^{*}, \\
Q_{k-1}^{0}(i) & :=Q_{k-1} \cap D_{k}(S)_{x_{i}, \delta}^{*}, \\
Q_{k-1}(i, A, B) & :=Q_{k-1}^{0}(i) \cap Q_{k-1}^{+}(A) \cap Q_{k-1}^{-}(B) .
\end{aligned}
$$

The sets $Q_{k-1}^{0}(i)$ and $Q_{k-1}^{-}(B)$ are compact, the other two are in general not. In view of (4.3), we have $Q_{k-1}=\bigcup_{(i, A, B)} Q_{k-1}(i, A, B)$, where the union runs over all partitions $\{1, \ldots, m\}=\{i\} \cup A \cup B$. Also, the sets $Q_{k-1}(i, A, B)$ form a partition of $Q_{k-1}(i)$ for a fixed $i$.

Step 6: Construction of a polynomial, which satisfies the assertion on $Q_{k-1}$. Again $\operatorname{ext} S=\left\{x_{0}, \ldots, x_{m}\right\}$, $G_{1}, \ldots, G_{s}$ are facets of $D_{k}(S)$ and $\delta$ is chosen as in Step 4. Moreover, by induction we constructed already polynomials $q_{i, k}, i=1, \ldots, m$. We choose $\rho>\delta$ such that $\left|l_{G_{j}}(x)\right| \leq \rho$ for all $x \in R_{k}, j=1, \ldots, s$. Consider the polynomial $\kappa=\kappa_{\delta, \rho, m} \in \mathbb{R}[t]$ as in Lemma 2.4 a). We set

$$
r_{i, k}:=q_{i, k} \prod_{\substack{j=1, \ldots, t \\ x_{i} \notin G_{j}}}\left(\kappa \circ l_{G_{j}}\right)^{n}
$$

and

$$
r_{k}:=\sum_{i=1}^{m} r_{i, k}
$$

where $n \in \mathbb{N}$ is to be fixed later. We claim the following.

Step 7: Verification that $r_{k}$ is a local solution. For sufficiently large $n$ one has:

a') $r_{k} \geq 0$ on $S$,

b') $r_{k} \leq 0$ on $Q_{k-1}$

c') $\left\{r_{k}=0\right\} \cap Q_{k-1} \subseteq S$.

Let us prove a'). We have $q_{i, k} \geq 0$ on $S$ for $i=1, \ldots, m$ and $\kappa \geq 0$ on $[-\rho, \rho]$, hence $r_{i, k} \geq 0$ on $S$ and finally $r_{k} \geq 0$ on $S$. In view of (4.3) we may replace $Q_{k-1}$ by $Q_{k-1}(i, A, B)$ for a given decomposition $\{1, \ldots, m\}=\{i\} \cup A \cup B$. Clearly, we have $r_{i, k} \leq 0$ on $Q_{k-1}^{0}(i)$ and $\left\{r_{i, k}=0\right\} \cap Q_{k-1}^{0}(i) \subseteq S$. Note that the factor $\prod_{\substack{j=1, \ldots, t \\ x_{i} \notin G_{j}}}\left(\kappa \circ l_{G_{j}}\right)^{n}$ is positive and arbitrarily large for sufficiently large $n$. Let

$$
r_{k, A}:=\sum_{\alpha \in A} r_{\alpha, k} \quad \text { and } \quad r_{k, B}:=\sum_{\beta \in B} r_{\beta, k},
$$

so $r_{k}=r_{i, k}+r_{A, k}+r_{B, k}$. We have $r_{A, k} \leq 0$ on $Q_{k-1}^{0}(i) \cap Q_{k-1}^{+}(A)$. So it is sufficient to show that $\left|r_{B, k}\right| \leq \frac{1}{2}\left|r_{i, k}\right|$ on $Q_{k-1}^{0}(i) \cap Q_{k-1}^{-}(B)$. For this it is enough to show that $\left|r_{\beta, k}\right| \leq \frac{1}{2 m}\left|r_{i, k}\right|$ on $Q_{k-1}^{0}(i) \backslash D_{k}(S)_{x_{\beta}}^{*}$ for all $\beta \in B$. We write

$$
Q_{k-1}^{0}(i) \backslash D_{k}(S)_{x_{\beta}}^{*}=\bigcup_{\substack{j=1, \ldots, t \\ x_{\beta} \notin G_{j}}}\left(Q_{k-1}^{0}(i) \cap\left\{l_{G_{j}} \leq 0\right\}\right) .
$$

So finally it remains to show that $\left|r_{\beta, k}\right| \leq \frac{1}{2 m}\left|r_{i, k}\right|$ on $Q_{k-1}^{0}(i) \cap\left\{l_{G_{j}} \leq 0\right\}$ for given $\beta, j$ and all sufficiently large $n \in \mathbb{N}$. Clearly $\left\{r_{i, k}=0\right\} \subseteq\left\{r_{\beta, k}=0\right\}$ and $q_{\beta, k}$ is bounded on this set. Moreover, by the properties of $\kappa$, we have

$$
\left|\prod_{\substack{j=1, \ldots, t \\ x_{\beta} \notin G_{j}}} l_{G_{j}}\right| \leq \frac{3}{4}
$$


Thus, the claim follows from the Hörmander-Łojasiewicz inequality (see Theorem 2.1).

Step 8: Conclusion. Let $T_{1}:=Q_{k-1}, T_{2}:=D_{k-1}(S) \backslash \operatorname{int}\left(R_{k}\right)$ and $h \in \mathbb{R}[X]$ be a non-negative polynomial which vanishes only on the Zariski closure of $S \cap T_{1}=\operatorname{ext}_{k} S$. Such a polynomial can easily be found with the additional property that $h>0$ on $T_{2}$ and for $x \in T_{2}: h(x) \rightarrow \infty$ as $\|x\| \rightarrow \infty$. Finally, let $f=r_{k}$. For $S, T_{1}, T_{2}, h, f$ we apply Proposition 2.11, which gives us a polynomial $p_{k}$ such that $p_{k} \geq r_{k}$ on $S, \operatorname{sign}\left(p_{k}\right) \leq \operatorname{sign}\left(r_{k}\right)$ on $Q_{k-1}$ and $p_{k}<0$ on $D_{k-1}(S) \backslash \operatorname{int}\left(R_{k}\right)$. That means $p_{k}$ satisfies a)-c).

As an immediate consequence of Proposition 4.2 we obtain

Corollary 4.3. Let $S \subseteq \mathbb{R}^{d}$ be a d-dimensional polytope. Then there are polynomials $p_{0}, \ldots, p_{d-1} \in \mathbb{R}[X]$ such that $S=\left\{p_{0} \geq 0, \ldots, p_{d-1} \geq 0\right\}$.

In the proof of Theorem 1.2 we shall use the following observation, see [GH03, Proposition 2.1] and also [BCR98, Section 6.5] or use an argument with fans as in [ABR96, Chapter VI, Section 7].

Proposition 4.4. Let $S$ be a d-polyhedron in $\mathbb{R}^{d}$, let $S=\left\{q_{1} \geq 0, \ldots, q_{m} \geq 0\right\}$ for $q_{1}, \ldots, q_{m} \in \mathbb{R}[X]$ and let $F$ be a face of $S$. Then at least $d-\operatorname{dim} F$ polynomials $q_{i}$ vanish on $F$. In particular, $m \geq d-\operatorname{dim} F$.

Proof of Theorem 1.2. Most arguments presented below are also given in $\mathrm{AH} 09 \mathrm{~b}$, but for the sake of completeness we give the whole proof.

We write $S=S_{0} \times \mathbb{R}^{k}$, where $\mathbb{R}^{d}=\mathbb{R}^{d-k} \times \mathbb{R}^{k}$ and $S_{0} \subseteq \mathbb{R}^{d-k}$ in suitable coordinates. We may assume that $k<d$, so $S$ admits at least one $k$-face, hence, by Proposition 4.4 $S$ cannot be described by fewer than $d-k$ polynomials.

Conversely, every polynomial description of $S_{0}$ easily extends to $S$. Note also, that $S_{0}$ does not contain any line. So it remains to show that a polyhedron $S \subseteq \mathbb{R}^{d}$ which does not contain any line is representable by $S=\left\{p_{0} \geq 0, \ldots, p_{d-1} \geq 0\right\}$ for suitable $p_{0}, \ldots, p_{d-1} \in \mathbb{R}[X]$.

For this we consider $\mathbb{R}^{d}$ as affine subspace $\left\{x \in \mathbb{R}^{d+1}: x_{d+1}=1\right\}$ in $\mathbb{R}^{d+1}$ and form the cone $C^{0}$ over $S$, that is $C^{0}:=\{\lambda a: a \in S, \lambda \geq 0\}$. The set $C:=\operatorname{cl} C^{0}$ is a polyhedral cone. Since $S$ does not contain a line, there is a linear form $l$ of $\mathbb{R}^{d+1}$ such that $C$ intersects the hyperplane $l=1$ properly, that is $S^{\prime}:=\{l=1\} \cap C$ is a polytope. By Corollary 4.3 we can find polynomials $q_{0}, \ldots, q_{d-1}$ such that $S^{\prime}=\left\{q_{0} \geq 0, \ldots, q_{d-1} \geq 0, l=1\right\}$. Possibly after an appropriate modification with the help of $l$, we assume that $q_{0}, \ldots, q_{d-1}$ are homogeneous and of even degree. We would like that $\left\{q_{0} \geq 0, \ldots, q_{d-1} \geq\right.$ $0\}=C \cup(-C)$, but this is possibly not true, since $\left\{q_{0} \geq 0, \ldots, q_{d-1} \geq 0, l=0\right\}$ may contain points other than $o$. In order to avoid the above situation we keep $q_{1}, \ldots, q_{d-1}$ as before and adjust $q_{0}$. We have $\left\{q_{0}=0\right\} \cap S=\operatorname{ext} S$ and $\left\{q_{0} \geq 0, l=1\right\}$ is compact. Then $q_{0}$ is negative semidefinite on $\{l=0\}$. We need to replace $q_{0}$ by a polynomial $p$ with $\{p \geq 0, l=0\}=\{o\}$. For this let $r_{1}, \ldots, r_{m}$ be (homogeneous) non-negative quadratic polynomials such that $\left\{r_{1}=0\right\}, \ldots,\left\{r_{m}=0\right\}$ are the affine hulls of the extremal rays of $C$, and let $r:=r_{1}, \ldots, r_{m}$. Now let

$$
p:=q_{0} l^{N-\operatorname{deg} q_{0}}-c r^{N},
$$

where $c>0$ is sufficiently small and $N \in \mathbb{N}$ is sufficiently large. Then $\{p \geq 0, l=0\}=\{o\}$ and $S \subseteq\{p \geq 0, l=1\}$ by the Hörmander-Lojasiewicz inequality. Hence

$$
\left\{p \geq 0, q_{1} \geq 0, \ldots, q_{d-1} \geq 0\right\}=C \cup(-C)
$$

Now we set $p_{0}\left(X_{1}, \ldots, X_{d}\right):=p\left(X_{1}, \ldots, X_{d}, 1\right)$ and $p_{i}\left(X_{1}, \ldots, X_{d}\right)=q_{i}\left(X_{1}, \ldots, X_{d}, 1\right)$ for $i=1, \ldots, d-$ 1 .

Remark 4.5. From the proofs we see that the polynomial representation $S=\left\{p_{0} \geq 0, \ldots, p_{d-1} \geq 0\right\}$ of a $d$-dimensional polytope $S$ that we construct above satisfies the condition

$$
\left\{p_{i}=0\right\} \cap S \subseteq \operatorname{ext}_{i} S
$$

for $i \in\{0, \ldots, d-1\}$. In fact, an arbitrarily representation of this size can easily be converted into a form satisfying the above properties. Let $S=\left\{q_{0} \geq 0, \ldots, q_{d-1} \geq 0\right\}$ be an arbitrary polynomial representation of a $d$-dimensional polyhedron $S$. For $p_{i}:=\sigma_{i+1}\left(q_{0}, \ldots, q_{d-1}\right)$ one has $S=\left\{p_{0} \geq 0, \ldots, p_{d-1} \geq 0\right\}$ (see Lemma 3.1). Furthermore, in view of Proposition 4.4, $p_{i}$ 's satisfy (4.4). 


\section{References}

[ABR96] C. Andradas, L. Bröcker, and J. M. Ruiz, Constructible Sets in Real Geometry, Ergebnisse der Mathematik und ihrer Grenzgebiete (3), vol. 33, Springer-Verlag, Berlin, 1996. MR 98e:14056

[AH09a] G. Averkov and M. Henk, Representing simple d-dimensional polytopes by d polynomials, Math. Program., Ser A. (2009).

[AH09b] Three-dimensional polyhedra can be described by three polynomial inequalities, Discrete Comput. Geom. 42 (2009), no. 2, 166-186. MR 2519874

[BCR98] J. Bochnak, M. Coste, and M.-F. Roy, Real Algebraic Geometry, Ergebnisse der Mathematik und ihrer Grenzgebiete (3), vol. 36, Springer-Verlag, Berlin, 1998. MR 2000a:14067

[Ber98] A. Bernig, Constructions for the theorem of Bröcker and Scheiderer, Master's Thesis, Universität Dortmund, pp. 48, 1998.

[BGH05] H. Bosse, M. Grötschel, and M. Henk, Polynomial inequalities representing polyhedra, Math. Program. 103 (2005), no. 1, Ser. A, 35-44. MR 2006k:52018

[Bos05] H. Bosse, Representing polyhedra by few polynomial inequalities, Dissertation, Technische Universität Berlin, 2005.

[Brö91] L. Bröcker, On basic semialgebraic sets, Exposition. Math. 9 (1991), no. 4, 289-334. MR 93b:14085

[GH03] M. Grötschel and M. Henk, The representation of polyhedra by polynomial inequalities, Discrete Comput. Geom. 29 (2003), no. 4, 485-504. MR 2004b:14098

[Hen07] M. Henk, Polynomdarstellungen von Polyedern, Jahresber. Deutsch. Math.-Verein. 109 (2007), no. 2, 51-69. MR 2340268

[Pau90] A. Pauluhn, Diploma thesis, 1990.

[Sch89] C. Scheiderer, Stability index of real varieties, Invent. Math. 97 (1989), no. 3, 467-483. MR $90 \mathrm{~g}: 14011$

[Zie95] G. M. Ziegler, Lectures on polytopes, Graduate Texts in Mathematics, vol. 152, Springer-Verlag, New York, 1995. MR 96a:52011 